\title{
Early clinical and biomechanical results following cervical arthroplasty
}

\author{
NeIl Duggal, M.D., M.Sc., F.R.C.S.(C), Gwynedd E. Pickett, M.D., F.R.C.S.(C), \\ Demytra K. Mitsis, and Jana L. Keller, M.Sc. (EPI) \\ Division of Neurosurgery, Department of Clinical Neurological Sciences, London Health Sciences \\ Centre, London, Ontario, Canada
}

\begin{abstract}
Object. Spinal arthroplasty may become the next gold standard for the treatment of degenerative cervical spine disease. This new modality must be studied rigorously to ensure in vivo efficacy and safety. The authors review the preliminary clinical experience and radiographic outcomes following insertion of the Bryan Cervical Disc Prosthesis (Medtronic Sofamor Danek, Memphis, TN).

Methods. This prospective cohort study included 26 patients undergoing single- or two-level implantation of the Bryan artificial cervical disc for treatment of cervical degenerative disc disease resulting in radiculopathy and/or myelopathy. Radiographic and clinical assessments were made preoperatively $1.5,3$ months, and at 6, 12, and up to 24 months postoperatively. The Neck Disability Index (NDI) and Short Form-36 (SF-36) questionnaires were used to assess pain and functional outcomes. Segmental sagittal rotation from C2-3 to C6-7 was measured using quantitative motion analysis software.

A total of 30 Bryan discs were placed in 26 patients. A single-level procedure was performed in 22 patients and a two-level procedure in the other four. Follow-up duration ranged from 1.5 to 27 months, with a mean duration of 12.3 months. A statistically significant improvement in the mean NDI scores was seen between pre- and late postoperative follow-up evaluations. A trend toward improvement in the SF-36 physical component was also found.

Motion was preserved in the treated spinal segments (mean range of motion $7.8^{\circ}$ ) for up to 24 months postsurgery. The relative contribution of each segment to overall spinal sagittal rotation differed depending on whether the disc was placed at C5-6 or C6-7. Overall cervical motion (C2-7) was moderately increased on late follow-up evaluations.

Conclusions. The Bryan artificial cervical disc provided in vivo functional spinal motion at the treated level. Overall cervical spinal motion was not significantly altered. Sagittal rotation did not change significantly at any level after surgery.
\end{abstract}

\section{KEY WORDS - range of motion - cervical spine • spinal arthroplasty • cervical disc prosthesis - artificial disc • clinical outcome}

Anterior cervical discectomy and fusion is a wellestablished, commonly performed surgical procedure for cervical spondylosis. Since its introduction by Robinson and Smith ${ }^{26,28}$ as well as Cloward ${ }^{6}$ in the 1950s, excellent clinical results have been reported in the treatment of degenerative disorders of the cervical spine..$^{3,5,16,20,21,28}$ Interbody fusion has the disadvantage, however, of converting a functionally mobile, mechanically stable spinal unit into a fixed, nonfunctional one. Analysis of strain distribution in intervertebral discs after ACDF has shown an increase in longitudinal strain, most commonly at levels immediately adjacent to the fused segment. ${ }^{22}$ The resulting increase in stress on discs adjacent to the fused level is thought to lead to accelerated disc degeneration and/or mechanical instability at adjacent levels. ${ }^{1,17,22,30}$ Radiographically confirmed changes consistent with spondylo-

Abbreviations used in this paper: $\mathrm{ACDF}=$ anterior cervical discectomy and fusion; $\mathrm{MR}=$ magnetic resonance; NDI = Neck Disability Index; ROM = range of motion; SF-36 = Short Form-36. sis and instability at levels above and below cervical fusions have been described by several authors, ${ }^{1,8,15,17,21,30}$ although these changes have not always been associated with recurrent clinical symptoms. ${ }^{4,9,19,23}$ There is controversy as to whether these changes are attributable to the biomechanical effects of fusion or are simply part of the natural history of spondylosis in a patient who is susceptible to cervical disc disease.

Cervical arthroplasty aims to restore normal spinal motion after anterior cervical discectomy and to avoid the abnormal kinematic stresses produced by anterior cervical fusion. The occurrence of adjacent-segment degeneration may be preventable if spinal motion can be maintained by means of a functional disc prosthesis. To achieve this goal, an artificial disc must mimic natural spinal kinematics as closely as possible, maintaining biomechanical parameters not only at the treated level but throughout the spine. Cervical disc prostheses must mimic the motion and elasticity of the natural disc, provide for balanced and integrated motion of the spinal unit, and possess maximum 
durability and biocompatibility. Their insertion should be safe, straightforward, and ideally should not add significantly to the duration of surgery. Finally, clinical success must match or surpass the excellent outcomes achieved using standard ACDF with or without plating.

We describe our early clinical and radiographic outcomes in a prospectively enrolled cohort of patients undergoing spinal arthroplasty with the Bryan Cervical Disc system (Medtronic Sofamor Danek, Memphis, TN). Quantitative motion analysis software was used to evaluate the in vivo sagittal rotation after cervical disc replacement.

\section{CLINICAL MATERIAL AND METHODS}

Since March 2002, information on clinical and radiographic outcomes after cervical arthroplasty has been collected prospectively. For this study we included all consecutive patients who underwent anterior cervical discectomy followed by insertion of the Bryan artificial cervical disc.

\section{Patient Population}

A total of 30 Bryan discs were placed in 26 patients between 2002 and 2004 (Fig. 1). At the time of surgery, the ages in our patient population ranged from 30 to 67 years, with a mean of $43.3 \pm 7.93$ years (mean \pm standard deviation) and included 16 men. A single-level procedure was performed in 22 patients and a two-level one in the other four. The duration of symptoms prior to cervical arthroplasty varied considerably between patients with radiculopathy and those with myelopathy. For patients with radiculopathy, the duration of preoperative symptoms ranged from 2.5 to 60 months (mean 12.5, median 8.5 months). In patients with myelopathy, preoperative symptoms had been present for 1 to 14 months prior to surgery (mean 6.2, median 5.5 months). The follow-up duration ranged from 1.5 to 27 months, with a mean of 12.3 months.

\section{Indications for Surgery}

Radiculopathy was present in 18 cases, myelopathy in six, and two patients presented with features of both. All patients received a specific diagnosis before they underwent surgery: 1) radiculopathy caused by soft disc herniation (10 patients); 2) radiculopathy caused by foraminal osteophytes (seven); 3) radiculopathy caused by soft disc herniation and foraminal osteophytes (one); 4) myelopathy caused by soft disc herniation (five); and 5) myelopathy caused by a hard disc (three). In all patients with radiculopathy, arm pain was their major symptom, comprising no less than $70 \%$ of their overall pain syndrome. Neck pain, to varying degrees, was present in $74 \%$ of patients. Four patients had previously undergone ACDF.

\section{Patient Selection Criteria}

Patients with cervical radiculopathy were considered for surgery only if they had experienced failure of nonoperative medical therapy, including activity modification, nonsteroidal antiinflammatory medications, physiotherapy, and massage. If mild-to-moderate asymptomatic degenerative changes were present adjacent to a symp-

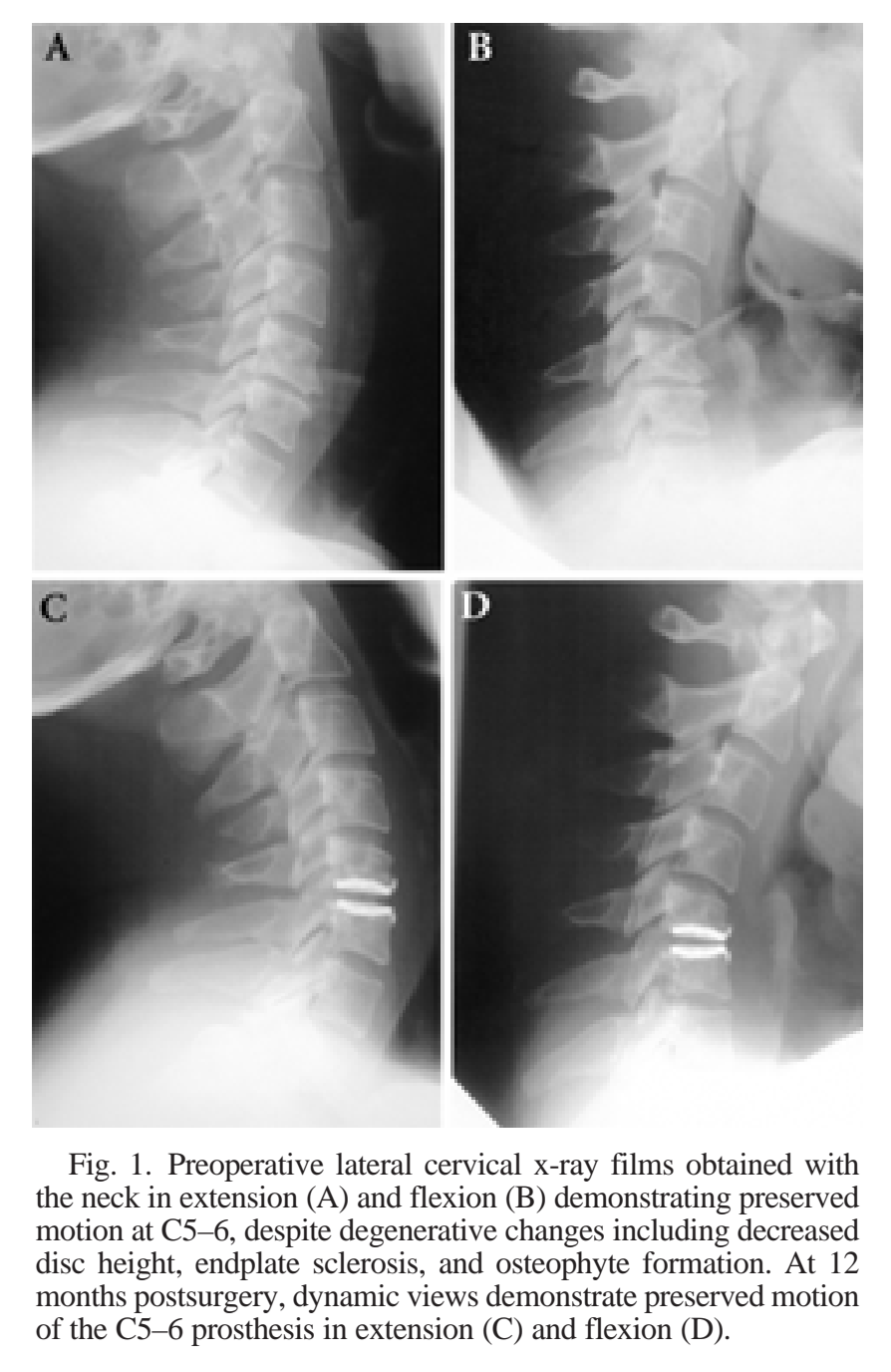

tomatic level, a single-level procedure was performed. Surgery was offered only to patients whose main symptom was arm pain, not neck pain. In patients presenting with spinal cord compression, the primary purpose of the surgery was to arrest progression of cervical myelopathy.

All patients were evaluated using static and dynamic cervical spine radiographs as well as computerized tomography scanning and MR imaging. Static neutral lateral radiographs were used to assess cervical sagittal balance, whereas anterior-posterior radiographs were used to exclude abnormal coronal alignment. Dynamic cervical spine radiographs were obtained to confirm motion at the level of the proposed surgery. Computerized tomography was used to assess the size of the disc prosthesis and was also valuable in visualizing the location of the foramen transversarium, the morphology of the vertebral body, the location of foraminal stenosis, and aided in the evaluation of facet disease. Preoperative MR imaging was performed in all patients to determine the origin of radiculopathy and/or myelopathy.

Surgery was offered to patients with a history and confirmatory findings on physical examination that were consistent with the observed imaging findings at the appropriate level. If the predominant symptom was neck pain, 
this was a contraindication for surgery. Patients were also excluded if there was a history of trauma, infection, or radiographic evidence of instability. Coexisting illnesses such as rheumatoid arthritis, renal failure, osteoporosis, and cancer as well as the use of preoperative corticosteroid medications were considered further exclusion criteria. Preoperative motion at the symptomatic level was a prerequisite for cervical arthroplasty. On the basis of those assessments, patients were grouped into the following categories: 1) soft disc herniation causing radiculopathy; 2) foraminal stenosis causing radiculopathy; 3) radiculopathy caused by soft disc herniation and foraminal osteophytes; 4) myelopathy caused by soft disc herniation; and 5) myelopathy caused by a hard disc. A subset of patients with myelopathy and radiculopathy presented with a history of ACDF. These patients developed symptomatic disease at a level adjacent or remote to the previous anterior cervical fusion.

\section{Data Collection}

For each eligible patient we prospectively collected information on sex, age, weight, and smoking status. The duration of preoperative symptoms, employment status, and return-to-work status were also recorded. In patients who had undergone prior procedures, the type and location of the previous surgery was noted.

Surgical Data. Operative details including the type of procedure (single- or two-level), duration of the surgery (including anesthetic time), blood loss, complications, the number of levels treated, the location of surgery, and the size of the device used were all recorded.

Clinical Outcomes Measures. All patients enrolled in this study underwent a complete neurological examination by the senior author prior to surgery and at each follow-up visit postoperatively. In addition to neurological outcomes, each patient completed surveys that measured pain and functional outcome. The NDI and SF-36 questionnaires were self-administered preoperatively and on the 1 st postoperative day as well as 1.5, 3, 6, 12, and 24 months postsurgery.

Radiographic Analysis. Static and dynamic cervical radiographs were obtained at $1.5,3,6,12$, and up to 24 months postoperatively.

In a subset of patients with at least 6 months of follow up, quantitative motion analysis software (Medical Metrics, Inc., Houston, TX) was used to analyze intervertebral motion. This validated radiographic motion analysis software uses advanced pattern-recognition algorithms to generate accurate measurements of segmental sagittal rotation (that is, ROM). ${ }^{25}$ The pre- and postoperative sagittal ROM in each spinal level was examined for three treated groups: patients with the prosthetic disc implanted at C5-6; those with the disc implanted at C6-7; and those with two-level procedures in which the disc was implanted at both C5-6 and C6-7. Time points for postoperative radiographic follow-up evaluations were defined as early (1.5-3 months) and late (6-24 months).

We defined the late follow-up period as 6 months or later, because we had found in a separate pilot study that there was no significant difference in sagittal ROM between 6 and 12 months of follow up (unpublished data). This likely reflects resolution of postsurgical neck and arm pain after 6 months, which may limit flexion and extension effort in earlier follow-up evaluations.

\section{Statistical Analysis}

We used descriptive statistics in the text and graphic representation to summarize clinical outcomes. For evaluation of the sagittal rotation, absolute values across groups were assessed, together with measures of change over time within individual patients. Parametric statistics include the Student two-sided t-test and analysis of variance, which were used to assess levels of statistical significance when comparing the continuous data.

\section{RESULTS}

All procedures were completed through a transverse skin incision made on the right side of the neck. The surgical technique has been described previously. ${ }^{13}$ The levels of surgery included the following: 1) C4-5 (one procedure); 2) C5-6 (13 procedures); and 3) C6-7 (16 procedures). The mean duration of surgery (including time for induction of anesthesia) was 3.1 hours. Blood loss ranged from 30 to $300 \mathrm{ml}$, with a mean loss of $106 \mathrm{ml}$. The median duration of hospitalization was 1.1 days (range $1-6$ days).

\section{Surgical Outcomes and Complications}

One patient experienced increased radicular pain in the recovery room following the procedure. An MR image obtained immediately postoperatively demonstrated adequate decompression of the nerve root, without residual soft disc. No new motor or sensory symptoms and signs were associated with this transient worsening of arm pain, which gradually improved over the next several weeks. Transient unilateral vocal cord paralysis, which resolved completely at 6 weeks, developed in another patient. Although there were no permanent esophageal or tracheal injuries, in a single case dysphagia persisted for almost 6 weeks postsurgery. No cerebrospinal fluid leaks or wound infections were encountered. No patient required revision surgery, and no prosthesis subsidence has been encountered. A single case of possible device migration was seen, in which the superior endplate of the disc had moved 2 $\mathrm{mm}$ anteriorly at 2 years postsurgery.

Four patients, three of whom had received plates, had undergone prior neck surgery (a two-level fusion had been performed in two cases). In all three patients with plates, the device was removed prior to placement of the artificial cervical disc. In three of the four patients a symptomatic disc herniation occurred adjacent to the earlier fusion. In a single case a C6-7 disc herniation occurred, causing myelopathy 10 years after a previous C4-5 ACDF. In this series cervical arthroplasty was not performed at the level of the previous surgery.

\section{Clinical Outcomes}

Outcome measurements for all patients were completed preoperatively and at regular intervals postoperatively. The distribution of mean pre- and postoperative SF-36 and NDI scores over time is shown in Figs. 2 and 3, respectively. A statistically significant improvement in the mean NDI scores was seen between preoperative scores 
(18.7) and late postoperative follow-up scores $(4.75 ; \mathrm{p}<$ $0.05)$. A trend toward improvement in the SF-36 physical component was found (Fig. 2). A similar trend toward improvement was seen in the mental component of the SF-36.

When patients suffering from a soft disc herniation were compared with those with foraminal stenosis, no statistically significant difference was found with respect to NDI and SF-36 outcome scores. Patients with a soft disc herniation had higher preoperative NDI scores (Fig. $4)$; the relative improvement in both groups was preserved. In addition, when outcomes in patients undergoing arthroplasty for myelopathy and/or radiculopathy were compared, no statistically significant difference was found.

\section{Employment Status}

Prior to surgery, 15 patients were working, 10 were receiving disability income, and two were homemakers. In the radiculopathy group (18 patients), all 11 individuals who were working preoperatively returned to work after treatment. Five patients with radiculopathy had been receiving disability payments preoperatively and four of them returned to work after surgery. In a single case, the patient did not return to work because of cardiac problems. Two patients who were receiving Workers' Compensation returned to work after surgery. For patients with radiculopathy, the time that elapsed before they returned to work ranged from 0.2 to 12 months (mean 3.2, median 2.8 months). In the myelopathy group (eight patients), all four who were working preoperatively returned to work. Three patients presented with an abrupt history of myelopathy and were admitted to the hospital, unable to work. Two of them were able to return to their previous employment. The homemaker who presented with myelopathy improved and was able to resume her prior activities. The range for elapsed time until return to work in the myelopathy group was 0.33 to 3 months (mean 2 , median 2.3 months).

\section{Radiographic Outcome}

A subset of patients underwent radiographic analysis. Eighteen discs were implanted in 16 patients: eight at C5-6 alone and six at C6-7 alone, whereas two patients underwent two-level implantation. The mean postoperative sagittal ROM at the treated disc space was $7.8^{\circ}$ on late

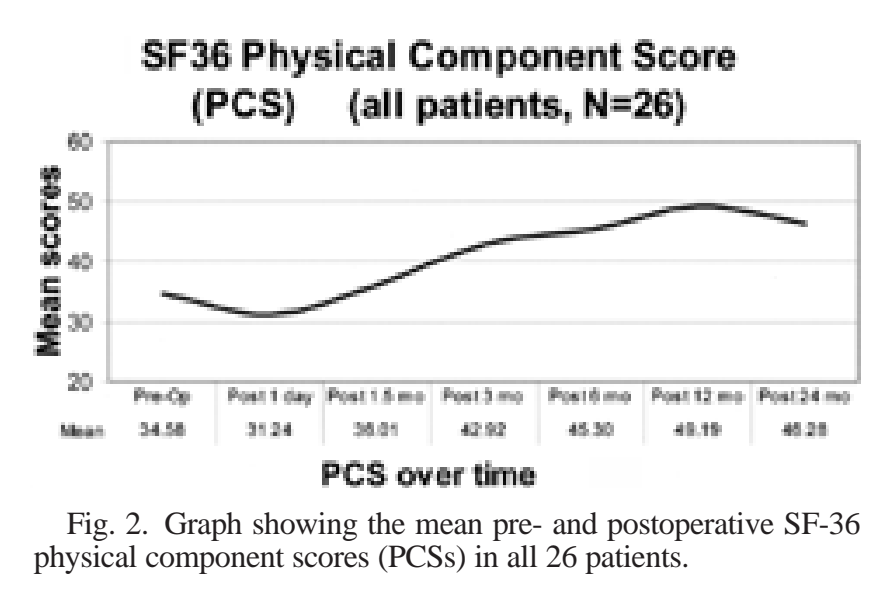
ent from the preoperative value of $10.1^{\circ}$. No significant change was observed in the mean ROM at adjacent or more distant spinal levels (Fig. 5). The amount of rotation contributed by each spinal level to the overall cervical sagittal rotation did not change significantly postsurgery. The overall cervical sagittal motion from C2-7 increased moderately in late follow-up evaluations (Fig. 6). This increase in mobility (mean $10.5^{\circ}$ ) was distributed over all spinal levels.

\section{DISCUSSION}

In this nonrandomized, prospective cohort study, we treated patients with cervical spondylosis with single- or two-level cervical disc replacement. Our preliminary results indicate that cervical arthroplasty provides favorable clinical and radiographic outcomes in this relatively short follow-up period. Our clinical results are in keeping with previously described clinical outcomes for cervical arthroplasty. ${ }^{13}$ In our small sample size, clinical outcomes in patients with radiculopathy were similar whether the cause was a soft disc herniation or osteophytes/foraminal stenosis. Radiographic results in this small group of patients demonstrated no significant change in sagittal rotation postsurgery. The relative contribution of each spinal segment to sagittal rotation was preserved following arthro-

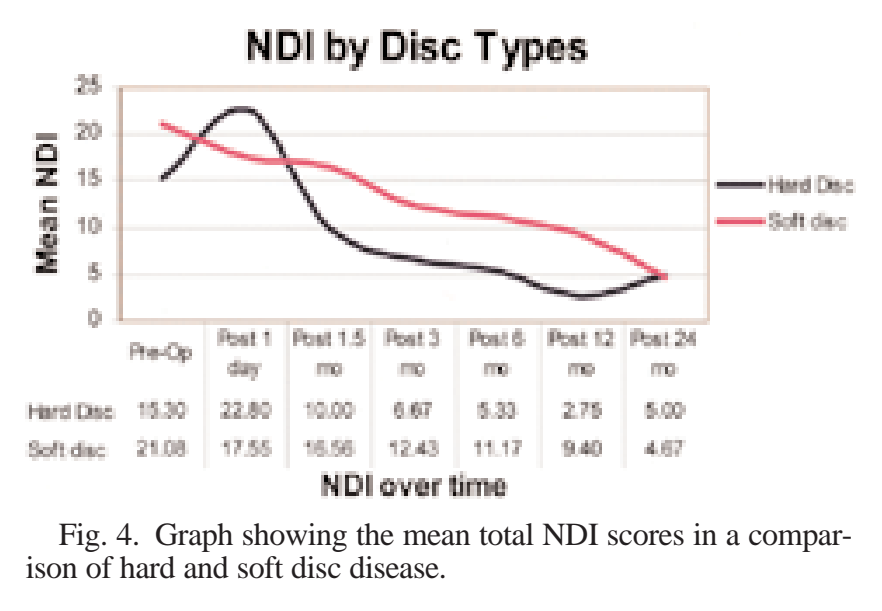




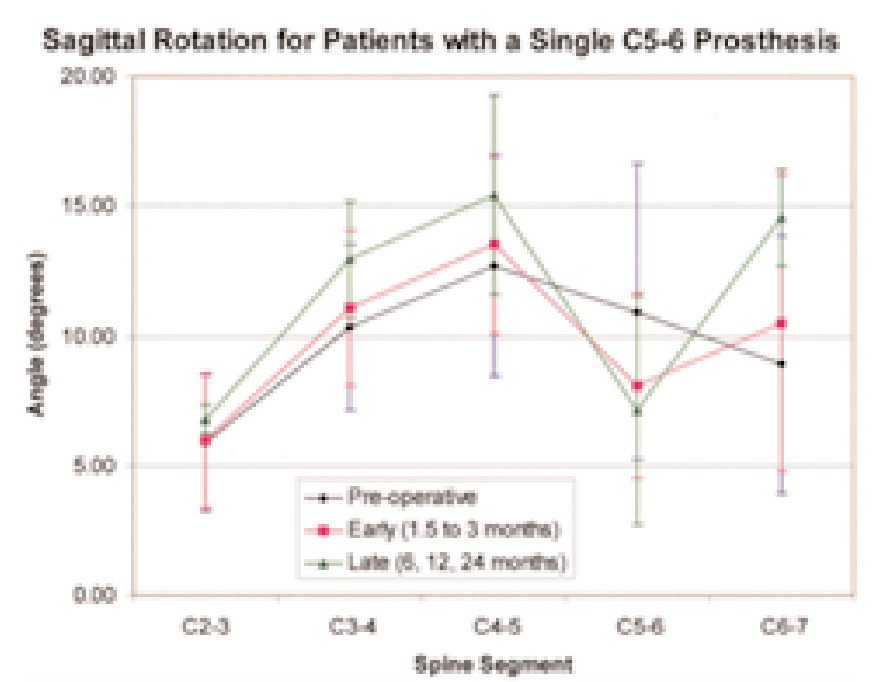

Sagittal Rotation for Patients with a Single C6.7 Prosthesis

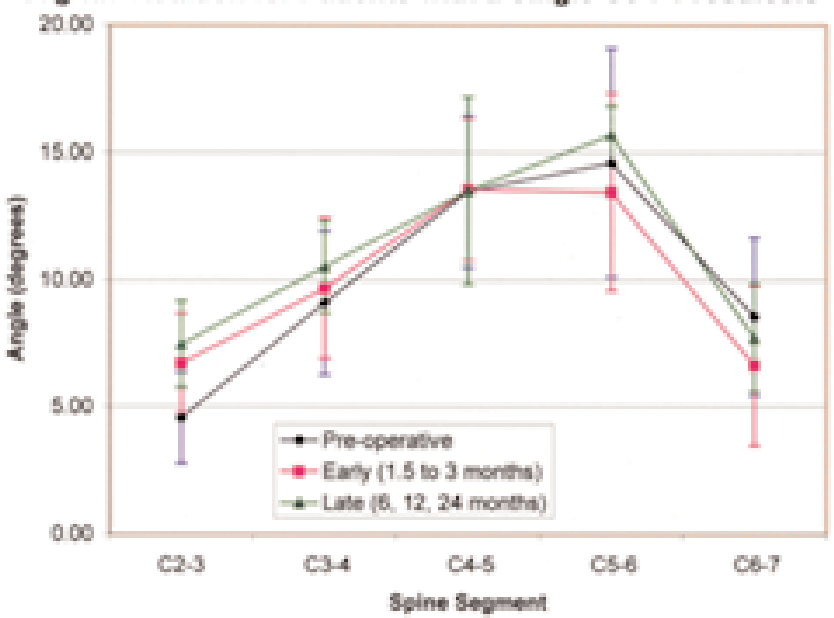

Fig. 5. Graphs showing the mean values for sagittal plane rotation preoperatively and in early and late follow-up evaluations. Upper: Rotation with disc implanted at C5-6. Lower: Rotation with disc implanted at C6-7.

plasty at C5-6 and/or C6-7. Patients exhibited a modest but significant increase in global cervical sagittal ROM in late follow-up evaluations compared with preoperative values.

\section{Literature Review}

Numerous authors have suggested that anterior cervical arthrodesis may alter the natural history of cervical spondylosis and hasten the development of degenerative changes at levels immediately above and below a fusion. ${ }^{4,9,12,16,17,29}$ Nevertheless, the concept of adjacent-segment disease, although widely discussed, remains unproven.

Patients who have undergone ACDF may display radiographic and clinical evidence of progressive degeneration. Long-term radiographic follow-up review in patients with anterior cervical fusion has demonstrated hypermobility and degenerative changes in the nonfused segments of the spine, including disc space narrowing, endplate sclerosis, and osteophyte formation. . $^{1,8,16,30,31}$ Reported rates of radi-

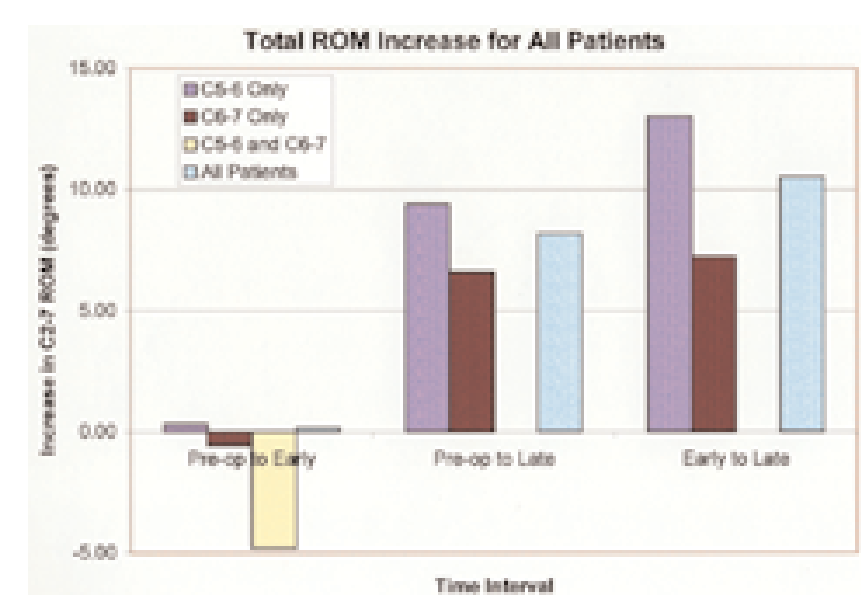

Fig. 6. Bar graph showing the change in cervical sagittal ROM (C2-7) experienced by all patients. An increase in overall spinal motion was identified only in late follow-up evaluations.

ographic evidence of degeneration adjacent to ACDF range from 0 to $81 \% \%^{8,14-16}$ and varies with the duration of follow up and inclusion of a control group. Although several authors have demonstrated radiographic degeneration with no clinical correlation, ${ }^{4,9,19,23}$ Gore and Sepic ${ }^{16}$ found an association between recurrent cervical pain and progression of spondylosis. Hilibrand, et al., ${ }^{17}$ performed a retrospective survivorship analysis of 409 ACDFs performed in 374 patients and found that symptoms of radiculopathy or myelopathy referable to an adjacent level occurred at a relatively constant rate of $2.9 \%$ per year, with a projected survivorship rate of $26 \%$ at 10 years. Again, however, the reported rates of symptomatic adjacent-segment disease vary widely, from 2.2 to $14 \%$ with varying follow-up durations. . $^{1,210,12,16,18}$

The true incidence of adjacent-segment disease remains a controversial issue. Degenerative changes occurring above and/or below a fused segment may simply represent the natural history of cervical spondylosis and may be related to genetic and physiological factors. In an analysis reported by Hilibrand, et al., ${ }^{17}$ patient age and evidence of preexisting degeneration had a higher degree of correlation with the risk of adjacent-segment disease than did the number of years since surgery. Clements and $\mathrm{O}^{\prime}$ Leary ${ }^{5}$ noted that recurrent clinical symptoms were more likely when asymptomatic spondylotic changes were present in the adjacent segments preoperatively.

Initial efforts at maintaining cervical motion after discectomy centered on placement of a ball-and-socket joint into the disc space; this device was secured to the adjacent vertebral bodies by anterior plates and screws. ${ }^{7,30}$ Complications associated with these devices include inadequate screw placement, screw failure, dysphagia due to the bulk of the anterior components, and joint subluxation. Nevertheless, cervical vertebral motion was preserved in the majority of cases, and most patients experienced improvement in their symptoms. At the 12-month follow-up review, patients who received an artificial cervical joint had an overall reduction in adjacent-level mobility, which was significantly different from the increased mobility observed in patients with ACDF. ${ }^{30}$ Pointillart ${ }^{24}$ published a report of the "first failure" of a cervical disc prosthesis; he 
reported that in eight of 10 patients, cervical mobility was not maintained at the implant level, and in the remaining two, in whom mobility was achieved, also experienced severe neck pain.

Favorable preliminary results have been reported with the use of the Bryan Cervical Disc Prosthesis in Europe. ${ }^{13}$ This prosthesis consists of a low-friction polyurethane nucleus surrounded by a polyurethane sheath, which is situated between two titanium alloy shells. The disc provides elasticity and compressibility, allowing for unconstrained motion and translation through the normal ROM. Early clinical results from European trials of single- and twolevel prosthesis implantation demonstrated that sagittal plane motion was preserved in $88 \%$ of patients treated with single-level and $86 \%$ of those with two-level prostheses at 1 year, and that clinical outcomes were rated as excellent, good, or fair in more than $90 \%$ of patients at 1 year. ${ }^{13}$

\section{Radiographic Outcomes}

It is clear that fusion alters spinal biomechanics. Anterior cervical arthrodesis increases the mechanical demand on the adjacent unfused spinal units, ${ }^{9}$ which may accelerate the rate of disc degeneration or mechanical instability. ${ }^{1,17,31}$ Biomechanical modeling of the cervical spine has led to predictions of increased intradiscal pressures at levels adjacent to intervertebral fusions. ${ }^{20}$ Adjacent-level motion has been shown to increase by $15 \%$ at 1 year after arthrodesis. ${ }^{31}$ This change is presumably caused by the fusion itself, rather than by other components of anterior cervical surgery such as elevation of the longus colli muscles. ${ }^{11}$ An increase in adjacent-level motion was not seen in patients who underwent anterior cervical discectomy followed by insertion of an artificial joint..$^{30}$

Analysis of the cervical ROM identified no significant change in sagittal rotation postsurgery in our patients. In addition, the degree to which each spinal segment contributed to spinal motion was unchanged. In particular, motion in the segments adjacent to the level of disc insertion was not significantly altered. Patients exhibited a modest but significant increase in global cervical sagittal motion on late follow-up evaluations, compared with preoperative values. With few exceptions, this increase in motion was distributed across all spinal levels, indicating that the cervical spine is more mobile after implantation of a single-level prosthesis. Although the cause of this general increase in mobility cannot be identified with certainty, we speculate that relief of neck pain by the time of late follow-up evaluations allowed for increased neck movement in these patients who had been symptomatic preoperatively.

\section{Clinical Outcomes}

Patients presenting with cervical radiculopathy and/or myelopathy, with radiological evidence of neural compression by osteophytic or herniated disc material, and with retained spinal motion at the level in question may be considered for placement of an artificial cervical disc. ${ }^{13,27}$ Our results demonstrated that patients suffering from hard or soft disc disease, myelopathy, or radiculopathy had improved NDI and SF-36 scores postsurgery. Nevertheless, these results are not surprising because the improve- ment in NDI and SF-36 scores is a function of the neural decompression. The potential benefit of arthroplasty over ACDF will be determined with the aid of late follow-up evaluations, in which the rates of adjacent-segment disease and repeated surgery are examined.

In our small cohort, patients with soft and hard disc disease demonstrated equally improved outcomes after surgery. Because the goal of cervical arthroplasty is to preserve motion, an aggressive decompression of hard discs must be undertaken during surgery to avoid residual symptoms. When a standard ACDF is performed for hard disc disease, the fusion and elimination of motion can promote bone remodeling and regression of osteophytes after surgery. With preserved motion following arthroplasty, however, the extent of the central and foraminal decompression is determined during surgery.

Our selection criteria for cervical arthroplasty continue to evolve as we gain more experience with this relatively new procedure. Young patients, who have a high lifetime risk of adjacent-segment disease, are suitable candidates for the prosthesis. Spinal arthroplasty may be particularly well suited for patients in whom there is an increased risk of adjacent-segment disease (that is, patients in whom asymptomatic spondylotic changes are present at adjacent or other spinal levels). In this group of patients, arthroplasty avoids incorporating multiple levels into the procedure. The artificial disc may also be indicated for patients who have previously undergone an ACDF and present with a new radiculopathy and/or myelopathy caused by a degenerated disc at an adjacent or distant level. All patients must undergo dynamic and static cervical radiographs before surgery. If motion is limited $\left(<3^{\circ}\right)$ or if there is evidence of advanced facet disease, these patients are excluded because they may not benefit from arthroplasty. There is little evidence that arthroplasty is beneficial when neck pain is the predominant symptom. Finally, in selecting patients for disc replacement, we choose those in whom a preserved cervical lordosis or mild straightening of the spine is found.

\section{Limitations of the Study}

The results of our study are not generalizable to all patients suffering from cervical disc disease. It is possible that our results have led us to overestimate the potential benefit of cervical disc replacement because of the subjective nature of the selection process, the small sample size, and the relatively short follow-up duration.

Unfortunately, because of power limitations, we were unable to conduct thorough analyses of clinical/radiographic factors predictive of success. Consequently, our negative results should be interpreted cautiously. Furthermore, the small sample size also limited our ability to develop a detailed protocol regarding patient selection. In future studies, a more rigorous characterization of the potential patient population is needed to help identify appropriate candidates and prognostic features for cervical arthroplasty. Randomization of surgical candidates with cervical disc disease to ACDF or cervical arthroplasty treatment groups will be essential in determining whether arthroplasty provides equivalent or improved outcomes and can be used to achieve the desired goal of preventing adjacent-segment disease. 
A major limitation in the assessment of preoperative imaging is that all patients in the study had symptomatic disc disease. The preoperative parameters observed therefore cannot be taken as "normal." Another major source of potential error in the radiographic analyses lies in the quality of flexion/extension imaging. Patient discomfort, lack of effort, out-of-plane motion, and the imaging method used may produce substantial variability in images obtained in the same patient at different time intervals. These factors can introduce important inaccuracies into the calculation of sagittal rotation. Every effort must be made to ensure consistency in the neuroimaging technique.

\section{CONCLUSIONS}

Insertion of the Bryan Cervical Disc Prosthesis following anterior cervical discectomy appears to be safe and provides good preliminary clinical results. Long-term follow-up review is required to assess its safety, efficacy, and ability to prevent adjacent-segment disease. Computerized analysis of the ROM demonstrated that the Bryan Cervical Disc Prosthesis provides in vivo functional spinal motion. The sagittal ROM was not significantly altered at any level after surgery. Overall cervical spinal motion from $\mathrm{C} 2-7$ increased moderately on late followup evaluations, with the increase in mobility distributed across all levels.

\section{References}

1. Baba $\mathrm{H}$, Furusawa $\mathrm{N}$, Imura $\mathrm{S}$, et al: Late radiographic findings after anterior cervical fusion for spondylotic myeloradiculopathy. Spine 18:2167-2173, 1993

2. Bohlman HH, Emery SE, Goodfellow DB, et al: Robinson anterior cervical discectomy and arthrodesis for cervical radiculopathy. Long-term follow-up of one hundred and twenty-two patients. J Bone Joint Surg Am 75:1298-1307, 1993

3. Bose B: Anterior cervical fusion using Caspar plating: analysis of results and review of the literature. Surg Neurol 49:25-31, 1998

4. Cherubino P, Benazzo F, Borromeo U, et al: Degenerative arthritis of the adjacent spinal joints following anterior cervical spinal fusion: clinicoradiologic and statistical correlations. Ital J Orthop Traumatol 16:533-543, 1990

5. Clements DH, O'Leary PF: Anterior cervical discectomy and fusion. Spine 15:1023-1025, 1990

6. Cloward RB: The anterior approach for removal of ruptured cervical discs. J Neurosurg 15:602-617, 1958

7. Cummins BH, Robertson JT, Gill SS: Surgical experience with an implanted artificial cervical joint. J Neurosurg 88:943-948, 1998

8. DePalma AF, Rothman RH, Lewinnek GE, et al: Anterior interbody fusion for severe cervical disc degeneration. Surg Gynecol Obstet 134:755-758, 1972

9. Döhler JR, Kahn MR, Hughes SP: Instability of the cervical spine after anterior interbody fusion. A study on its incidence and clinical significance in 21 patients. Arch Orthop Trauma Surg 104:247-250, 1985

10. Emery SE, Bohlman HH, Bolesta MJ, et al: Anterior cervical decompression and arthrodesis for the treatment of cervical spondylotic myelopathy. Two to seventeen-year follow-up. J Bone Joint Surg Am 80:941-951, 1998

11. Fuller DA, Kirkpatrick JS, Emery SE, et al: A kinematic study of the cervical spine before and after segmental arthrodesis. Spine 23:1649-1656, 1998

12. Geisler FH, Caspar W, Pitzen T, et al: Reoperation in patients after anterior cervical plate stabilization in degenerative disease. Spine 23:911-920, 1998

13. Goffin J, Van Calenbergh V, van Loon J, et al: Intermediate follow-up after treatment of degenerative disc disease with the Bryan Cervical Disc Prosthesis: single-level and bi-level. Spine 28:2673-2678, 2003

14. Goffin J, van Loon J, Van Calenbergh F, et al: Long-term results after anterior cervical fusion and osteosynthetic stabilization for fractures and/or dislocations of the cervical spine. $\mathbf{J}$ Spinal Disord 8:499-508, 1995

15. Gore DR, Gardner GM, Sepic SB, et al: Roentgenographic findings following anterior cervical fusion. Skeletal Radiol 15: 556-559, 1986

16. Gore DR, Sepic SB: Anterior cervical fusion for degenerated or protruded discs: a review of one hundred forty-six patients. Spine 9:667-671, 1984

17. Hilibrand AS, Carlson GD, Palumbo MA, et al: Radiculopathy and myelopathy at segments adjacent to the site of a previous anterior cervical arthrodesis. J Bone Joint Surg Am 81: 519-528, 1999

18. Hilibrand AS, Yoo JU, Carlson GD, et al: The success of anterior cervical arthrodesis adjacent to a previous fusion. Spine 22:1574-1579, 1997

19. Hunter LY, Braunstein EM, Bailey RW: Radiographic changes following anterior cervical fusion. Spine 5:399-401, 1980

20. Jacobs B, Krueger EG, Leivy DM: Cervical spondylosis with radiculopathy. Results of anterior diskectomy and interbody fusion. JAMA 211:2135-2139, 1970

21. Lunsford LD, Bissonette DJ, Janetta PJ, et al: Anterior surgery for cervical disc disease. Part 1: Treatment of lateral cervical disc herniation in 253 cases. J Neurosurg 53:1-11, 1980

22. Matsunaga S, Kabayama S, Yamamoto T, et al: Strain on intervertebral discs after anterior cervical decompression and fusion. Spine 24:670-675, 1999

23. McGrory BJ, Klassen RA: Arthrodesis of the cervical spine for fractures and dislocations in children and adolescents. A longterm follow-up study. J Bone Joint Surg Am 76:1606-1616, 1994

24. Pointillart V: Cervical disc prosthesis in humans: first failure. Spine 26:E90-E92, 2001

25. Reitman CA, Hipp JA Nguyen L, et al: Changes in segmental intervertebral motion adjacent to cervical arthrodesis: a prospective study. Spine 29:E221-E226, 2004

26. Robinson RA, Smith GW: Anterolateral cervical disc removal and interbody fusion for cervical disc syndrome. Bull Johns Hopkins Hosp 96:223-224, 1955

27. Sekhon LHS: Cervical arthroplasty in the management of spondylotic myelopathy. J Spinal Disord Tech 16:307-313, 2003

28. Smith GW, Robinson RA: The treatment of certain cervicalspine disorders by anterior removal of the intervertebral disc and interbody fusion. J Bone Joint Surg Am 40:607-623, 1958

29. Truumees E, Herkowitz HN: Adjacent segment degeneration in the cervical spine: incidence and management. Semin Spine Surg 11:373-383, 1999

30. Wigfield C, Gill S, Nelson R, et al: Influence of an artificial cervical joint compared with fusion on adjacent-level motion in the treatment of degenerative cervical disc disease. J Neurosurg (Spine 1) 96:17-21, 2002

31. Wu W, Thuomas KA, Hedlund R, et al: Degenerative changes following anterior cervical discectomy and fusion evaluated by fast spin-echo MR imaging. Acta Radiol 37:614-617, 1996

Manuscript received July 16, 2004.

Accepted in final form August 16, 2004.

Address reprint requests to: Neil Duggal, M.D., Division of Neurosurgery, Department of Clinical Neurological Sciences, London Health Sciences Centre, 339 Windermere Road, London, Ontario, Canada N6A 5A5. 